\title{
Comparing Glucose Concentration Among Six Saliva Collection Methods In The Newly Developed Saliva Glucose Detection System
}

Cui Yangyang ( $\nabla$ cuiyy20@mail.tsinghua.edu.cn )

Tsinghua University

Zhang Hangkun

Tsinghua University

Zhu Jia

Tsinghua University

Peng Lu

Tsinghua University

Duan Zhili

Tsinghua University

Liu Tian

Tsinghua University

Zuo Jiasheng

Tsinghua University

Xing Lu

Tsinghua University

Liao Zhenhua

Tsinghua University

Wang Song

Tsinghua University

Liu Weiqiang

Tsinghua University

\section{Research Article}

Keywords: Saliva, Glucose, Methods, Diabetes mellitus, Sample collection

Posted Date: July 21st, 2021

DOI: https://doi.org/10.21203/rs.3.rs-731252/v1 
License: (c) (i) This work is licensed under a Creative Commons Attribution 4.0 International License. Read Full License 


\section{Abstract}

Objective: This study aims to identify the ideal saliva collection technique and propose a newly sensitive approach to achieve daily non-invasive blood glucose monitoring for healthy people.

Method: A total amount of six methods were employed for collecting saliva glucose in 20 healthy participants in the morning (9:00), before lunch (11:00), after lunch (14:00) and in the evening (17:00). Ultra-micro ultraviolet spectrophotometer was used to launch a new saliva glucose detection system in measuring the saliva glucose concentration.

Results: The newly developed glucose detection system showed a statistically significant linear relationship in the range of 0.1-6 mg/dl with $R^{2}=0.999$. Besides, the parotid saliva glucose concentration was higher than both the sublingual/submandibular saliva and the whole saliva. The parotid glucose concentration fluctuating after meal, and the sublingual/submandibular glucose concentration steady.

Conclusions: The detection limit was 1/110 of the average blood glucose concentration of healthy people when fasting, which fully met the sensitivity requirements for detecting the saliva glucose concentration of healthy people. Besides, the collection method was an important factor that affected the saliva glucose concentration. The unstimulated parotid saliva glucose may be easier to reflect blood glucose concentration, which provided a reference for the evaluation of the health status of subjects and the early prediction of diabetes mellitus.

\section{Introduction}

Diabetes mellitus (DM) is a globally common chronic disease affecting humans, which remains one of the major health concerns in the 21st century [1]. Without urgent and sufficient actions, it is predicted that 578 million people will have DM in 2030 and the number will increase by $51 \%$ reached 700 million in $2045[2,3]$. Meanwhile, with the gradual improvement of people's awareness of disease prevention, it is very necessary to predict the health status of healthy people and predict the disease through the monitoring of daily physiological indicators.

Blood glucose measurement is an indispensable method for screening and controlling DM. However, routine blood glucose detection requires invasive venipuncture or acupuncture, which brings pain to the patient and affects the patient's enthusiasm for blood glucose monitoring. Therefore, non-invasive blood glucose monitoring has attracted great attention [4, 5]. Among the most non-invasive methods, saliva glucose in replacement of blood glucose imposes a very positive significance in monitoring the conditions. This research area has already generated a plethora of previous scholarly works $[6,7]$. Caixeta et al. [8] showed that saliva was a promising result for the detection and monitoring of DM, and proved the accuracy of laboratory testing of saliva glucose was $95.2 \%$. Rodrigue et al. [9] pointed out that saliva like blood can reflect the changes in human physiological functions, so it may be a substitute for early detection and monitoring of DM. Meanwhile, saliva collection is convenient, safe, non-invasive, no risk of 
infection and painless to patients. Therefore, people pay more and more attention to it in experimental research and clinical use [10].

Although saliva glucose reflects the health of the human body, its use as a diagnostic fluid has been hindered and neglected, mainly because of the lack of standardized saliva collection techniques and comprehensive detection methods for the study of saliva glucose. Most studies use saliva as a diagnosis with different collection methods and often lack to define characterization of the patients or clear sampling processes [11,12]. This makes it difficult to compare the results of different studies [13]. In general, most studies view saliva wrongly as a homogeneous body fluid. However, saliva is not a solitary fluid and cannot be viewed as such. Instead, it is a complex mixture consisting of the secretions of the three main glands (parotid, submandibular, and sublingual), each of which secretes a characteristic type of saliva, hundreds of small salivary glands, gingival crevicular fluids and debris. It is also unstable, but constantly changing, and its composition is affected by other things such as sampling method, environment, oral hygiene, psychological status and general health [14]. As a diagnostic medium, saliva also has some disadvantages which needs to be considered. For example, the concentration of molecules in saliva is extremely low, and the low glucose concentration in saliva is only $1 / 100$ to $1 / 50$ of the blood glucose concentration. Therefore, the study of highly sensitive glucose detection methods is one of the key technologies to achieve non-invasive detection $[15,16]$. Secondly, because many factors affect saliva secretion and composition, it is necessary to establish precise standards for saliva collection [17]. Such as the type of saliva glucose, that is, saliva produced by whole saliva or specific glands, and whether the sample was collected after stimulation. [18, 19]. Besides, a plethora of literature works focused on the patients with DM. Rarely has been studied when the participants are healthy people, because there has been no detection system so far [19].

This study aims to to identify the ideal saliva collection technique and develop new sensitive method to achieve daily non-invasive blood glucose monitoring for healthy people. Ultra-micro ultraviolet spectrophotometer was used to realize a new saliva glucose detection system. The optimal $\mathrm{pH}$ and ionic strength of the reaction system was screened, and the glucose oxidase method with 2,4,6-tribromo-3hydroxybenzoic acid (TBAHA) as the chromogen was improved. Six saliva collection methods were used to collect saliva glucose in 20 healthy people in the morning (9:00), before lunch (11:00), after lunch (14:00) and in the evening (17:00). Then the developed glucose detection system was used to measure the saliva glucose concentration.

\section{Materials And Methods}

\subsection{Participants}

In this study, 20 healthy participants with a mean \pm SD age of $27.5 \pm 4.1$ years were included. Inclusion criteria were good general health, age $\geq 18$ years, and body mass index $(\mathrm{BMI}) \leq 30 \mathrm{~kg} / \mathrm{m}^{2}$. All the participants were free of fever or cold and maintain exceptional oral hygiene on the day of collection. If oral examination indicated poor oral hygiene, hyposalivation, oral complaints, or some other oral 
diseases (e. g. mucosal lesions, clinical signs of ongoing periodontal diseases), they were directly excluded from further involvement in the study. All participants signed an informed consent form.

\subsection{Glucose collection}

Avoid smoking, brushing teeth, eating or drinking 30 minutes before collection. Then the mouth was rinsed with water before collection to remove food residues in the oral cavity [20]. Salivette (Sarstedt, 51.5134) (including untreated swabs and swabs stimulated by citric acid) was used to collect saliva glucose, including six collection methods.

For each participant, samples of parotid, sublingual/submandibular, and whole saliva were collected with and without stimulation (as shown in Fig. 1). Respectively denoted as unstimulated whole saliva (UWS), stimulated whole saliva (SWS), stimulated parotid saliva (SPS), unstimulated parotid saliva (UPS), unstimulated sublingual/submandibular saliva (USS), stimulated sublingual/submandibular saliva (SSS).

All saliva glucose were collected in the same clinical room and at the same time in the four time periods of 9:00-9:30, 11:00-11:30, 14:00-14:30 and 17:00-17:30. After completing the above steps, the cotton swab soaked was spit with saliva back into the collection tube, then the saliva was collected and weighed with electronic-balance (denoted as $Q_{1}$ after centrifugation [21], and it was frozen directly at $-20^{\circ} \mathrm{C}$ for testing after collection [22]. The saliva flow rate (SFR) reflected the amount of saliva, and calculated as in Eq. (1).

$\mathrm{SFR}=\frac{Q_{1}-Q_{2}}{T}$

SFR was the saliva flow rate, $Q_{1}$ was the gross weight, $Q_{2}$ was the weight of collection tube, $T$ was the collecting time.

\subsection{Glucose analyses}

The optimal $\mathrm{pH}$ and ionic strength of the reaction system were screened, and the glucose oxidase method using TBHBA as the chromogen was improved. Accurately $20 \mathrm{mg}$ of glucose was weighed to dissolve in artificial saliva (Phygene, $\mathrm{PH}=7$, China), which was more than $99 \%$ similar to the saliva secreted by the real human body, then it was transferred to a $100 \mathrm{~mL}$ volumetric flask, which was diluted to the mark to obtain a $20 \mathrm{mg} / \mathrm{dl}$ artificial saliva glucose standard solution. Finally, it was diluted to 30 concentrations between $0.1-6 \mathrm{mg} / \mathrm{dl}$, which were used for calibration of artificial saliva glucose solution measurement. Meanwhile, $0.25 \mathrm{mkat} / \mathrm{LGOD}$ and $0.17 \mathrm{mkat} / \mathrm{LPOD}$ as $1 \mathrm{mg} / \mathrm{mL}$ stock solutions according to the product instructions were prepared, and they were stored in aliquots and freeze at $-20^{\circ} \mathrm{C}$. An appropriate amount was taken for each experiment and diluted to the required concentration with PBS. 4-aminoantibiotic Bilene $(0.5 \mathrm{mmol} / \mathrm{L})$ and TBHBA $(5 \mathrm{~g} / \mathrm{L})$ were prepared by dissolving in PBS. 
Ultra-micro ultraviolet spectrophotometer (Nano-Drop One Microvolume,Thermo Fisher Scientific $\square$ America) was used to detect the wavelength of the sample. The wavelengths were selected at $500,505,510,515,520,525,530,535 \mathrm{~nm}$ to detect the absorbance (ABS) value of the same sample, and the most relevant wavelength was selected. PBS with PH values of 5.6, 5.9, 6.2, 6.5, 6.8, 7.1, 7.4, 7.7, 8 were prepared. $0.1,0.5,0.9,1.3,1.7$ and $2 \mathrm{mg} / \mathrm{dl}$ glucose sample were added to detection systems with different PH values, the correlations were calculated. PBS $(\mathrm{pH}=6.5)$ with $\mathrm{NaCl}$ concentration of $1,3,5,7$, $9,11,13,15,17 \mathrm{mg} / \mathrm{dl}$ were prepared. The sample group was set to $0.5,1,1.5 \mathrm{mg} / \mathrm{dl}$ glucose sample, and the correlations were calculated.

The newly constructed system was used to determine the linear range of glucose concentration detection using a newly constructed $\mathrm{pH} 6.5, \mathrm{NaCl}$ concentration of $5 \mathrm{mg} / \mathrm{dl}$ reaction system to detect the ABS value of a glucose standard solution with a concentration of $0.1-6 \mathrm{mg} / \mathrm{dl}$, and the results obtained were drawn into a standard curve, and the method was used to measure the collected saliva samples.

\subsection{Statistics}

SPSS was used to perform statistical analysis. The data were expressed as relative numbers, and $\chi^{2}$ was used for comparison between groups. The measurement data was conformed to the normal distribution and expressed as mean \pm standard deviation $(\overline{\mathrm{x}} \pm \mathrm{s})$, and the t-test was used for comparison between groups. The Shapiro-Wilk test was used to test the normality of sample data. Non-normally dis- tributed data were described in terms of minimum and maximum numbers, and normally distributed data were

described in terms of $(\bar{x} \pm s)$. Besides, hypothesis testing would have insufficient sensitivity when the sample size was small, which would cause the results to lose use value, and if the data deviates slightly from normality, the final test result would not have much impact, so box plots and qq plots could also be combined to perform statistical analysis. $\mathrm{P}<0.05$ indicated that the difference was statistically significant.

\section{Results}

\subsection{Saliva detection method}

The reaction product of the glucose concentration detection system had the highest $A B S$ value measured at 510 and $520 \mathrm{~nm}$ wavelengths. When $\mathrm{PH}=6.5$, the $A B S$ correlation was highest $\left(\mathrm{R}^{2}=0.9948\right)$ at $520 \mathrm{~nm}$ wavelength (as shown in Fig. 2). Comprehensively $520 \mathrm{~nm}$ of the maximum absorption wavelength and the PH 6.5 were chosen.

The correlations between different $\mathrm{NaCl}$ concentrations and $\mathrm{ABS}$ when the glucose concentrations were $0.5 \mathrm{mg} / \mathrm{dl}, 1 \mathrm{mg} / \mathrm{dl}$ and $1.5 \mathrm{mg} / \mathrm{dl}$ were shown in Table S1 (as shown in the supplementary material). When $\mathrm{NaCl}$ were $5 \mathrm{mg} / \mathrm{dl}$ and $9 \mathrm{mg} / \mathrm{dl}$, the correlations were the highest at $0.999,0.999$, but the Sy. $\mathrm{x}$ was the smallest at $5 \mathrm{mg} / \mathrm{dl}$, and the correlation decreased when it was higher or lower than $5 \mathrm{mg} / \mathrm{dl}$. Therefore, the optimal ion environmental concentration was selected as $5 \mathrm{mg} / \mathrm{dl}$. 
The glucose concentration was taken as the $x$ axis and the ABS as the $y$ axis, linear regression was performed to obtain the standard curve equation as $y=1.68 x+0.04, R^{2}=0.999$, as shown in Fig. 3 . It showed that the newly constructed system had a good linear relationship with the detected ABS value when the glucose concentration was in the range of $0.1-6 \mathrm{mg} / \mathrm{dl}$. The lower limit of this range was $0.1 \mathrm{mg} / \mathrm{dl}$ which was $1 / 110$ of the average blood glucose concentration of healthy people when fasting, which fully met the sensitivity requirements for detecting the saliva glucose concentration of healthy people.

\subsection{Sample characteristics}

Table S2 (as shown in the supplementary material) was saliva glucose levels of the studied groups, the SFR in the UPS was the smallest, followed by the USS, and the largest was UWS. Besides, stimulation of citric acid could increase the SFR. The SFR at 14:00 was the lowest.

Figure 4. was the summary of saliva data. Figure 4(a) was ABS of saliva glucose concentration at different times and different saliva collection methods. It could be seen that the saliva concentration of UPS was significantly higher than the other five methods. At the same time, the stimulated saliva glucose concentration was significantly lower than the unstimulated saliva glucose concentration. In general, the amount of saliva collected by stimulated method was much greater than that of the unstimulated method, but the stimulated saliva glucose concentration was lower than the unstimulated saliva glucose concentration.

It showed the trends of saliva glucose concentration with different saliva collection methods at different times in Fig. 4(b). It could be seen that the largest saliva glucose concentration in the morning (9:00). UWS, UPS, USS, SWS, SSS had the same trend (Fall, rise, fall). In general, different saliva collection methods had different trends in saliva glucose concentration, which showed that the saliva collection method had a great influence on the saliva glucose concentration.

\subsection{The normal distribution curve of each collection method}

Figure 5 was the normal distribution curve of different time and different collection methods. The normal distribution curve reflected the distribution law of random variables, which indicated the potential of the data for saliva glucose testing. e. g. In Fig. 5(b), it was the qq-plot in 11:00, SSS, USS and UPS had the better distribution law of random variables, SIG. were $0.155,0.065$ and 0.141 respectively. So, it could be seen that UPS in 9:00 (as shown in Fig. 5(a)), SSS, USS and UPS in 11:00 (as shown in Fig. 5(b)),SPS, UPS and USS in 14:00 (as shown in Fig. 5(c)), UPS, SPS and SSS in 17:00 (as shown in Fig. 5(d)) had good normal distribution. In general, UPS had better normal distribution than other saliva collection methods in most time periods. So parotid glucose may be better used to respond to saliva glucose. In general, UPS had the highest glucose concentration and better normal distribution, higher than UWS, and higher than USS.

\subsection{Changes in saliva glucose before and after meals}


Figure 6 was the changes of saliva glucose before and after meals by different saliva collection methods. It could be seen that different saliva collection methods had a great influence on the concentration of saliva glucose. The saliva glucose concentration collected by UPS, USS, and SPS were greater after meals than before meals, while the saliva glucose concentration collected by SWS, SPS and SSS were not changed much before and after meals. At the same time, it could also be seen that some people had higher saliva glucose concentration before meals than after meals, which might be related to eating habits and digestive ability.

\subsection{Correlation between different saliva collection methods}

Table S3 (as shown in the supplementary material) was the correlation between different saliva collection methods, it could be seen that UWS/SWS in $14: 00$ was the most relevant $(p=0.676)$. Secondly were USS/SSS in 9:00, and USS/SSS in 11:00 and 14:00. At the same time, it was found that there was no obvious correlation between saliva collection methods at other times.

\section{Discussion}

Currently, non-invasive detection techniques based on saliva samples are basically targeted at DM patients. With the change of living habits, the proportion of people facing hidden dangers of DM will increase. Therefore, the importance and necessity of routine blood glucose monitoring for healthy people is self-evident. In this study we constructed a saliva-based high-sensitivity glucose detection system, and this method was used to compare the SFR and saliva glucose concentration of six different saliva collection methods for the first time.

The research of non-invasive blood glucose detection methods is one of the hot topics of great concern in the international medical and sensor fields. It will make a qualitative leap in the detection of DM and has important practical significance for the treatment of DM. Therefore, there are many people engaged in this research at home and abroad, but due to various reasons, still various defects in various detection methods, which need to be improved and developed. Besides, commercial instruments have not yet been launched. E. g. in the optical rotation method, the components such as lithium tantalum crystals used therein are greatly affected by temperature, so the environmental temperature is relatively high, which brings inconvenience to household use [23]. Ryman spectroscopy analysis method: the structure is relatively complex, and it is not easy to be accepted by patients [24]. Ion chromatography analysis method: its sensitivity, stability and accuracy are poor, and this method is susceptible to interference from other substances in saliva, which affects the detection results [25]. The ultra-micro ultraviolet spectrophotometer method in this study is a highly repeatable method with high sensitivity and high detection accuracy.

In this study, a new sensitive method was developed to realize daily non-invasive blood glucose monitoring of healthy people. An automated analysis method using TBHBA as the chromogen was developed for the detection of saliva glucose. This method not only increased the amount of sample, but also used a highly sensitive chromogen. This study found that the product has a maximum absorption at 
a wavelength of $520 \mathrm{~nm}$, and the optimal $\mathrm{pH}$ is 6.5 , the optimal $\mathrm{NaCl}$ concentration is $5 \mathrm{mg} / \mathrm{dl}$. The environmental $\mathrm{PH}$ value will change or affect the dissociation state of the enzyme and the substrate to increase or decrease the enzyme activity. Therefore, the maximum activity of the enzyme required the corresponding optimum PH value. The main catalytic enzymes in the test solution are GOD and POD. The activity of the former was in the range of $\mathrm{pH} 4.0$ to 7.0, and the activity of the latter was in the range of $\mathrm{pH} 5.0$ to 9.0. The optimal $\mathrm{PH}$ value of the reaction test solution measured in the experiment was 6.5, which was within the range of GOD activity and POD activity. Therefore, it was theoretically speculated that the $\mathrm{PH}$ value of 6.5 was the comprehensive optimal $\mathrm{PH}$ value of the same system where the two enzymes were located. Besides, it is found that its linear range, accuracy, and precision can meet the requirements of detection, and more importantly, it makes the measurement process more standardized, reduces errors, and is simple and easy to implement.

Saliva, like plasma or serum, is a unique and complex body fluid. Sufficient saliva secretion is essential for maintaining oral health. The advantages of saliva assessment include the cost-effectiveness of noninvasive collection and screening of large populations [26]. Saliva is currently considered to be an excellent diagnostic biomarker for human characteristics. Although in this study, no research on the correlation between salivary glucose concentration and blood glucose concentration was done, a large number of scholars' studies have demonstrated the potential of salivary glucose in blood glucose detection. For example, the saliva microbiome is considered to be as accurate as fingerprints in terms of biometrics when used to screen for normality of characteristics [27]. In some laboratory tests, saliva may be used as a substitute for blood, for example, to detect viral infections, DM[28, 29]. Some scholars have different conclusions, which are mainly caused by different saliva collection methods and time. The 6 saliva collection methods and 4 saliva collection times studied in this study can make up for the shortcomings of existing research and provide the next step for the concentration of glucose in saliva. The determination of saliva has laid a good foundation and pointed out the direction for finding the most suitable method and time to collect saliva glucose. Therefore, it can better improve the accuracy of saliva glucose detection and provide a theoretical basis for non-invasive blood glucose detection in saliva.

The determination of saliva glucose concentration is a prerequisite for the development of saliva as a diagnostic and prognostic tool for DM biomarker discovery. In this case, it is important to keep the technical variability caused by sample collection and processing to a minimum so that inter-subject variability in health and disease states can be assessed reproducibly [30]. Single or mixed saliva can be collected. It should be noted that many unknown factors and unstable elements will affect the properties of mixed saliva. Saliva collected directly from a single gland is stable and not affected by oral conditions. Thus, it can accurately reflect blood glucose status. Saliva from the parotid gland is easily collected under unstimulated and stimulated conditions. Dhanya et al. [31] reported that when saliva is collected under unstimulated conditions, the concentration of glucose in saliva is higher than under stimulated conditions, which is consistent with the conclusions obtained in this study. Other studies have found that there is no significant difference in the concentration of glucose in saliva collected under unstimulated and stimulated conditions [32]. Because the participants may not be easy to accept acid stimulation, and 
the water concentration in stimulated saliva is higher. Besides, unstimulated saliva may be more representative of a normal physiological state.

Takeda et al. [33] measured the saliva chemical concentration of healthy subjects under different conditions and found that compared with stimulated saliva, almost all metabolites in unstimulated saliva were higher. Jha et al. [34] also found that compared with stimulated saliva, the average saliva glucose level in unstimulated saliva of control and non-control DM patients was higher. Saliva collected directly from a single gland is stable and not affected by oral conditions. Therefore, it may accurately reflect blood glucose status. In this study, six saliva collection methods were used include whole saliva, parotid saliva, and sublingual/submandibular saliva in stimulated and unstimulated states. It was found that the glucose concentration of unstimulated saliva was higher than that of stimulated saliva. Secondly, the UPS glucose was higher than the USS glucose and higher than the UWS glucose.

The relative contribution of different glands to the whole saliva varies with the collection method, the degree of stimulation, age and even the time of day [35]. The variable nature of saliva secretion suggests that different methods may have to be used when studying its components or their possible role as indicators of specific physiological conditions. There is a large amount of literature on the diagnostic possibilities of saliva, but there is still no standardized technique for collecting saliva samples. In different studies, different sampling methods are often used, and many studies do not or rarely describe patient preparation or sampling procedures [36]. In addition, without proper clinical examination, the characteristics of participants are usually insufficient. Most saliva glucose research papers focus on studying the whole saliva [37] because it can be easily obtained by spitting it into a test tube or letting it drip from the mouth. Few people pay attention to ductal saliva obtained from different salivary glands [38]. Moreover, as far as we know, this is the first study focused on structurally comparing the glucose expression of whole saliva and glandular saliva in a cohort of careful characterization and clinical examination. The results indicate that different collection methods provide significant differences in the snapshots of saliva glucose.

Our results revealed a close correlation between the SPS and UPS, as well as the USS and UWS glucose. The main function of the parotid gland is to digest carbohydrates and form food masses, so the serous cells dominate, making the glands mainly secrete serous secretions with high glucose concentration. This may explain the high glucose concentration in parotid saliva. The main function of sublingual saliva is to lubricate the oral cavity and prevent chemical and mechanical influences. Therefore, there is little change before and after meals.

The limitation of our study is the relatively small sample size. Further studies with a larger sample size are necessary to confirm the correlation between blood glucose and saliva glucose, so as to design a saliva-based diagnostic test method for DM. In addition, there are still many problems in this study that need to be resolved and further explored. For example, the submandibular glands and sublingual glands are closely located, so it is difficult to separate saliva from these glands with certainty, which is why 
saliva is collected from both glands in the current study. How to distinguish sublingual saliva from submandibular saliva is also a direction that needs further research.

In summary, the results of this study indicate that different saliva collection methods provide significant differences in the snapshots of saliva glucose. Based on the comparison of unstimulated and stimulated saliva collection methods, it can be shown that based on the simplicity and low variability of the collection method, UPS may be a preferred collection method. The results emphasize the importance of consistency when collecting saliva samples, which should be more important than the collection method itself.

\section{Conclusion}

In this study, ultra-micro ultraviolet spectrophotometer was used to realize a new saliva glucose detection system, which laid a good foundation for realizing blood glucose detection without trauma. The lower limit of the concentration range determined in this study was $0.1 \mathrm{mg} / \mathrm{dl}$, and the lower limit of detection was $1 / 110$ of the average concentration of blood glucose in healthy people when fasting, which fully met the sensitivity requirements for detecting the concentration of saliva glucose in healthy people. The collection method was an important factor that affected the saliva glucose concentration and SFR. The stimulated method was easy to collect saliva, and the saliva amount was large and could meet the needs of saliva glucose detection, but the concentration was lower, the parotid saliva concentration was the highest, and the postprandial change was large. The UPS glucose might be easier to replace blood to reflect blood glucose concentration, which provided a reference for the evaluation of the health status of subjects and the early prediction of DM.

\section{Declarations}

\section{Acknowledgments}

This project was supported by the Guangdong Basic and Applied Basic Research Foundation (Grant No. 2020B1515120082), the Innovation Commission of Science and Technology of Shenzhen Municipality (Grant No. JCYJ20190807144001746, Grant No. JSGG20191129114422849) and the Shenzhen Municipal Industrial and Information Technology Bureau (Grant No. 20180309163834680).

\section{Ethics declarations}

\section{Conflict of interest}

The authors declare that they have no conflict of interest.

\section{Consent statement/ethical approval}

All study subjects signed an informed consent form, and the study had been approved by the Beijing Ethics Committee. 


\section{References}

1. Gautam S, Upadhyay A, Mutha R, et al. Potential role of darvyadi kwatha in the management of diabetes[J]. 2021,12(1):1-7.

2. Pouya S, Inga P, Paraskevi S, et al. Global and regional diabetes prevalence estimates for 2019 and projections for 2030 and 2045: Results from the International Diabetes Federation Diabetes Atlas, 9 th edition[J]. Diabetes Research and Clinical Practice, 2019,157: 107843.

3. Jovanovi J, Mihailovi M, Uskokovi A, et al. The Effects of Major Mushroom Bioactive Compounds on Mechanisms That Control Blood Glucose Level[J]. Journal of Fungi - Open Access Mycology Journal, 2021, 7(1):58-65.

4. Baghelani M, Abbasi Z, Daneshmand $M$, et al. Non-invasive continuous-time glucose monitoring system using a chipless printable sensor based on split ring microwave resonators[J]. Scientific Reports, 2020, 10(1):12980.

5. Meyhfer S, Wilms B, F Ihling, et al. Evaluation of a near-infrared light ultrasound system as a noninvasive blood glucose monitoring device[J]. Diabetes, Obesity and Metabolism, 2020, 22(4): 694698.

6. Mohammadnejad P, AsI S S, Aminzadeh S, et al. A new sensitive spectrophotometric method for determination of saliva and blood glucose[J]. Spectrochimica Acta Part A Molecular and Biomolecular Spectroscopy, 2019, 229:117897.

7. Singh A K, Jha S K. Fabrication and validation of a handheld non-invasive, optical biosensor for selfmonitoring of glucose using saliva[J]. IEEE Sensors Journal, 2019, 19(18):1-10.

8. Caixeta D C, Aguiar E, Cardoso-Sousa L, et al. Salivary molecular spectroscopy: a rapid and noninvasive monitoring tool for diabetes mellitus during insulin treatment[J]. PLoS One,2019, 15(3)区 e0223461.

9. Rodrigues R, Vieira W, Siqueira W L, et al. Saliva as a tool for monitoring hemodialysis: a systematic review and meta-analysis[J]. Brazilian Oral Research, 2021, 35(8): e016.

10. Lee L T, Wong Y K, Hsiao H Y, et al. Evaluation of saliva and plasma cytokine biomarkers in patients with oral squamous cell carcinoma - ScienceDirect[J]. International Journal of Oral and Maxillofacial Surgery, 2018, 47(6):699-707.

11. Kodintsev A N, Kovtun O P, Volkova L I. Saliva biomarkers in diagnostics of early stages of Alzheimer's disease[J]. Neurochemical Journal, 2020, 14(4):429-438.

12. Petruczynik A, K Wróblewski, Wojtanowski $K$, et al. Comparison of various chromatographic systems for identification of vortioxetine in bulk drug substance, human serum, saliva, and urine samples by HPLC-DAD and LC-QTOF-MS[J]. Molecules, 2020, 25(11):2483.

13. Li Y, Li R, Li X, et al. Effects of different aerobic exercise training on glycemia in patients with type 2 diabetes: A protocol for systematic review and meta analysis [J]. Medicine, 2021, 100(18): e25615.

14. Almeida Pdel V, Gregio A M, Machado M A, et al. Saliva composition and functions: a comprehensive review[J]. The journal of contemporary dental practice,2008, 9:72-80. 
15. Sagana M, Dharman S. Estimation of salivary and blood glucose level among patients with diabetes mellitus - A comparative study[J]. Journal of Pharmaceutical Research International, 2020,10(6):2027.

16. Kothai G, Alex A, Patil A B, et al. Correlation of salivary glucose level with blood glucose level in patients with diabetes mellitus[J]. International Journal of Research in Pharmaceutical Sciences, 2020, 11(4):1410-1414.

17. Lee, Kwon, Shin, et al. Optimization of Saliva Collection and Immunochromatographic Detection of Salivary Pepsin for Point-of-Care Testing of Laryngopharyngeal Reflux[J]. Sensors, 2020, 20(1):325333.

18. Kai D T, Kenny L, Frazer I H, et al. High-risk human papillomavirus detection in oropharyngeal cancers: Comparison of saliva sampling methods[J]. Head \& Neck, 2019, 41: 1484-1489.

19. Bellagambi F G, Lomonaco T, Salvo P, et al. Saliva sampling: Methods and devices. An overview[J]. TrAC Trends in Analytical Chemistry, 2019, 124:115781.

20. Ceccato F, Barbot M, Albiger N, et al. Daily salivary cortisol and cortisone rhythm in patients with adrenal incidentaloma [J]. Endocrine, 2018, 59(3): 510囚519.

21. Ng CM, Lam TK, Au Yeung YC, et al. Clinical utility of late冈night and post囚overnight dexamethasone suppression salivary cortisone for the investigation of Cushing's syndrome [J]. Hong Kong Med J, 2017, 23(6):570ه578.

22. Mészáros K, Karvaly G, Márta Z, et al. Diagnostic performance of a newly developed salivary cortisol and cortisone measurement using an LC囚MS / MS method with simple and rapid sample preparation[J]. J Endocrinol Invest, 2018, 41(3):315区323.

23. Yu S, Zhao Q, He Y, et al. Thermodynamic study on the experiment of measuring the rate constant of sucrose hydrolysis reaction by optical rotation method[J]. University Chemistry, 2021, 36(4): 2005064

24. Mukasheva A, Saparkhojayev N, Akanov Z, et al. The prevalence of diabetes in the republic of kazakhstan based on regression analysis methods[J]. International Journal of Health and Medical Sciences, 2020, 24(15) \8232-8238.

25. Chaila M Z, Viniegra M, Gagliardino J J, et al. Glycated hemoglobin measurement: comparison of three methods versus high performance liquid chromatography[J]. Journal of Diabetes Science and Technology, 2021:193229682199717.

26. Ghizoni J S, Nichele R, Oliveira M, et al. The utilization of saliva as an early diagnostic tool for oral cancer: microRNA as a biomarker[J]. Clinical and Translational Oncology, 2019, 22(6): 804-812.

27. M R Parisi, Soldini G D, Perri S, et al. Offer of rapid testing and alternative biological samples as practical tools to implement HIV screening programs[J]. New Microbiologica, 2009,32(4):391-396.

28. Sanchez B M, Luna R F, R Mendoza G, et al. A machine-learning strategy to evaluate the use of FTIR spectra of saliva for a good control of type 2 diabetes[J]. Talanta, 2020, 221(1):121650.

29. Peter, Hettegger, Jasmin, et al. High similarity of $\lg G$ antibody profiles in blood and saliva opens opportunities for saliva-based serology[J]. PloS one, 2019, 14(6): 0218456. 
30. Al Kawas S, Rahim Z H, Ferguson D B, et al. Potential uses of human salivary protein and peptide analysis in the diagnosis of disease[J]. Archives of oral biology,2012, 57(1):1-9.

31. M Dhanya, Hegde, Salivary glucose as a diagnostic tool in Type II diabetes mellitus: a case-control study[J]. Nigerian Journal of Clinical Practice, 2016,19(4):486-490, 2016.

32. Vuletic $L$, Spalj $S$, Rogic $D$, et al. The rise in glucose concentration in saliva samples mixed with test foods monitored using a glucometer: An observational pilot study[J]. Journal of Oral Biosciences, 2019, 61(4):201-206.

33. Takeda I, Stretch C, Barnaby P, et al. Understanding the human salivary metabolome[J]. NMR in Biomedicine, 2010, 22(6):577-584.

34. Jha. S K, David. C M, Saluja. I P, et al. Estimation of salivary glucose level and plasma glucose level in subjects with and without diabetes mellitus: a comparative study[J]. National Journal of Integrated Research in Medicine, 2013, 5(3):65-70.

35. Jasim H, Carlsson A, Hedenberg-Magnusson B, et al. Saliva as a medium to detect and measure biomarkers related to pain[J]. Scientific Reports, 2018, 8(1):3220.

36. Hu S, Loo J A, Wong D T, et al. Human saliva proteome analysis and disease biomarker discovery[J]. Expert review of proteomics, 2007,4:531-538.

37. Jayapiriya U S, Goel S. Flexible and optimized carbon paste electrodes for direct electron transferbased glucose biofuel cell fed by various physiological fluids[J]. Applied Nanoscience, 2020, 10(11):1-10.

38. Denny, P. et al. The proteomes of human parotid and submandibular/sublingual gland salivas collected as the ductal secretions[J]. Journal of proteome research,2008,7:1994-2006.

\section{Figures}




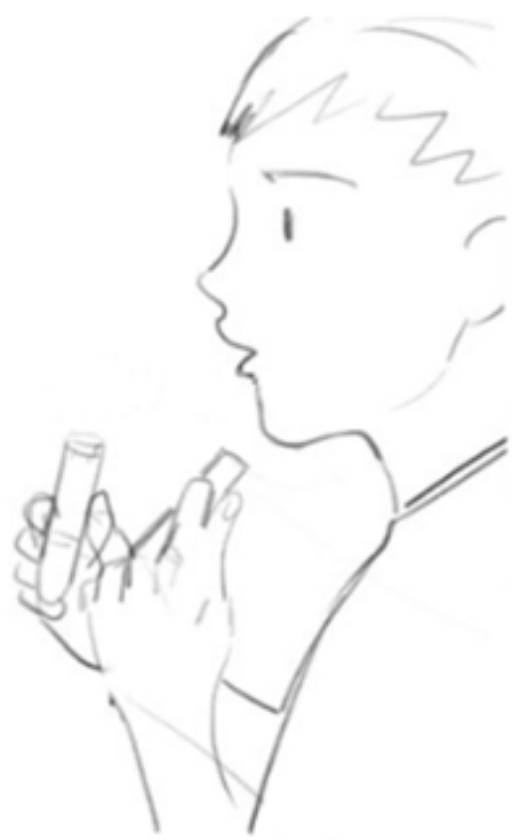

(a)

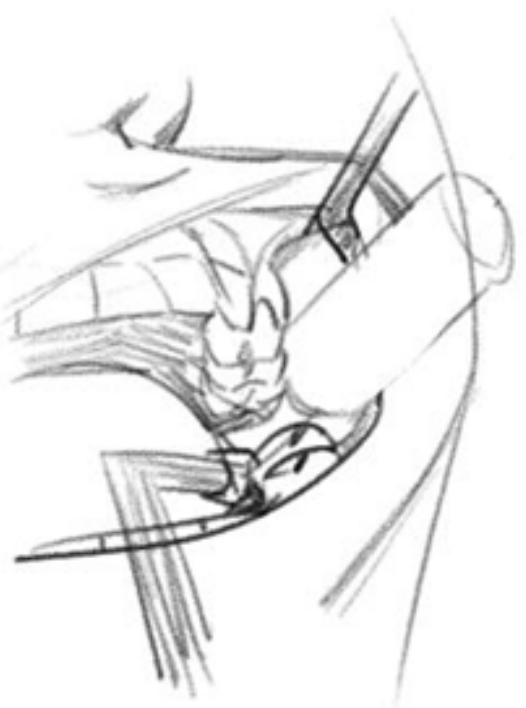

(b)

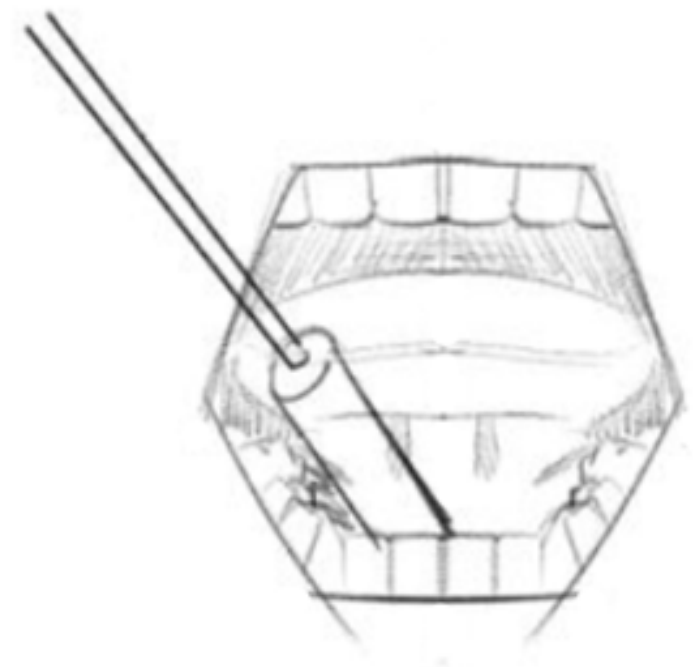

(c)

\section{Figure 1}

Six methods for collecting glucose (a) UWS/SWS: the swab in the test tube was taken out and put in the mouth to chew for $1 \mathrm{~min}$ (b) UPS/SPS: the swab was placed near the left parotid duct and it was taken out after 5 mins(c) USS /SSS: the swab was put under the tongue and it was taken out after 3 mins

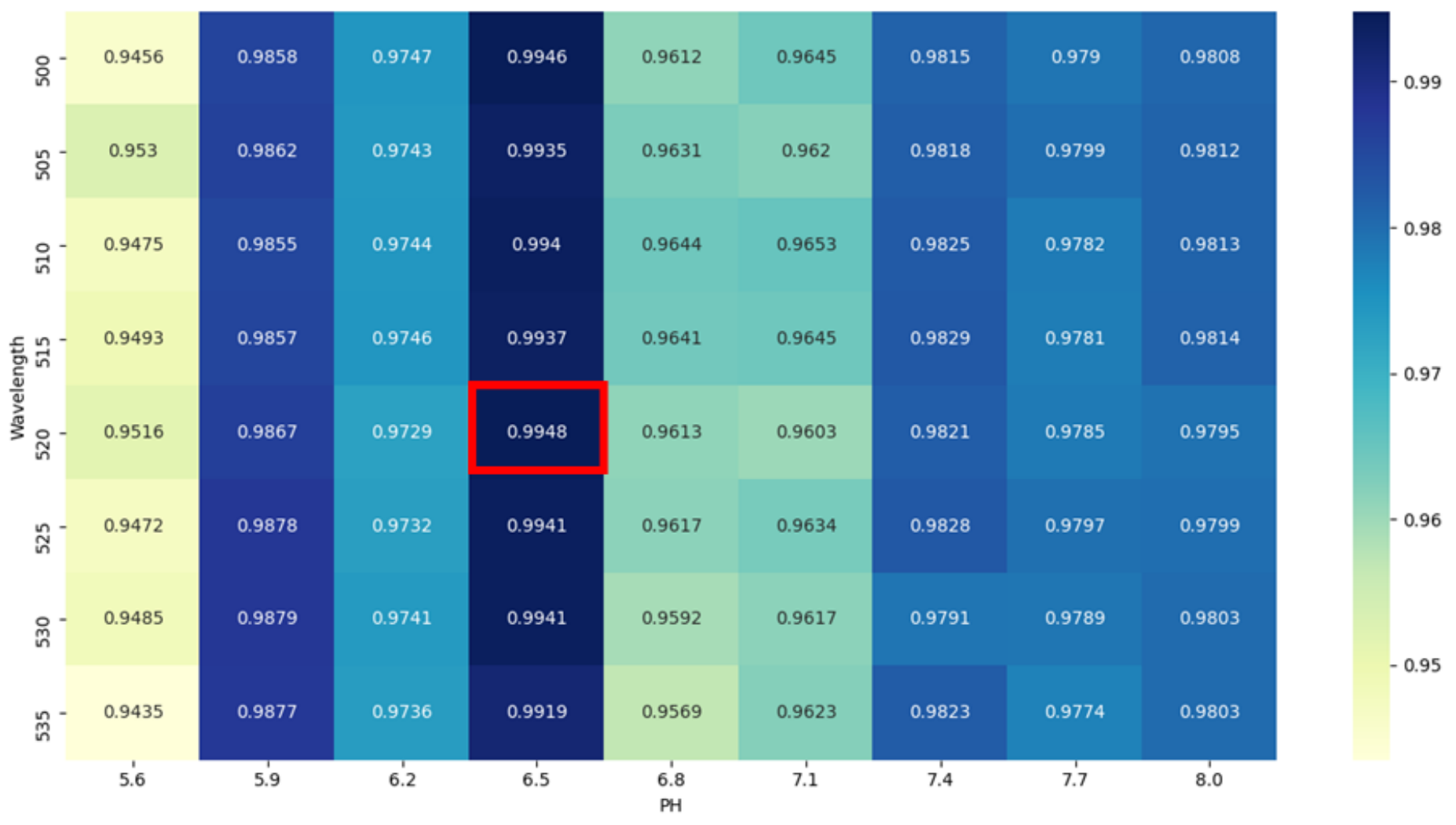

Figure 2 
The influence of wavelength and $\mathrm{pH}$ on the ABS of the sample.

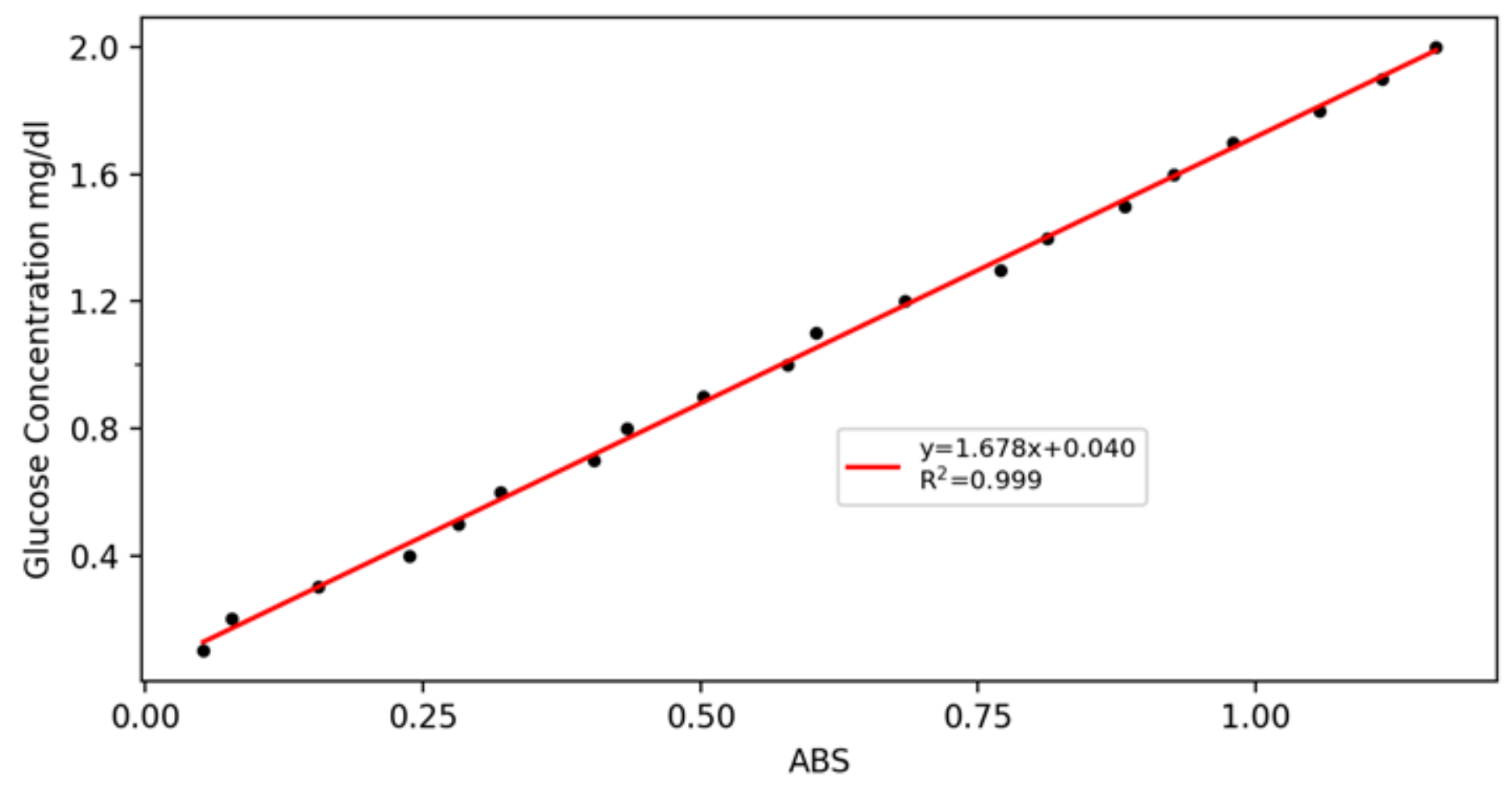

Figure 3

Standard curve of ABS values of different concentrations of glucose. 


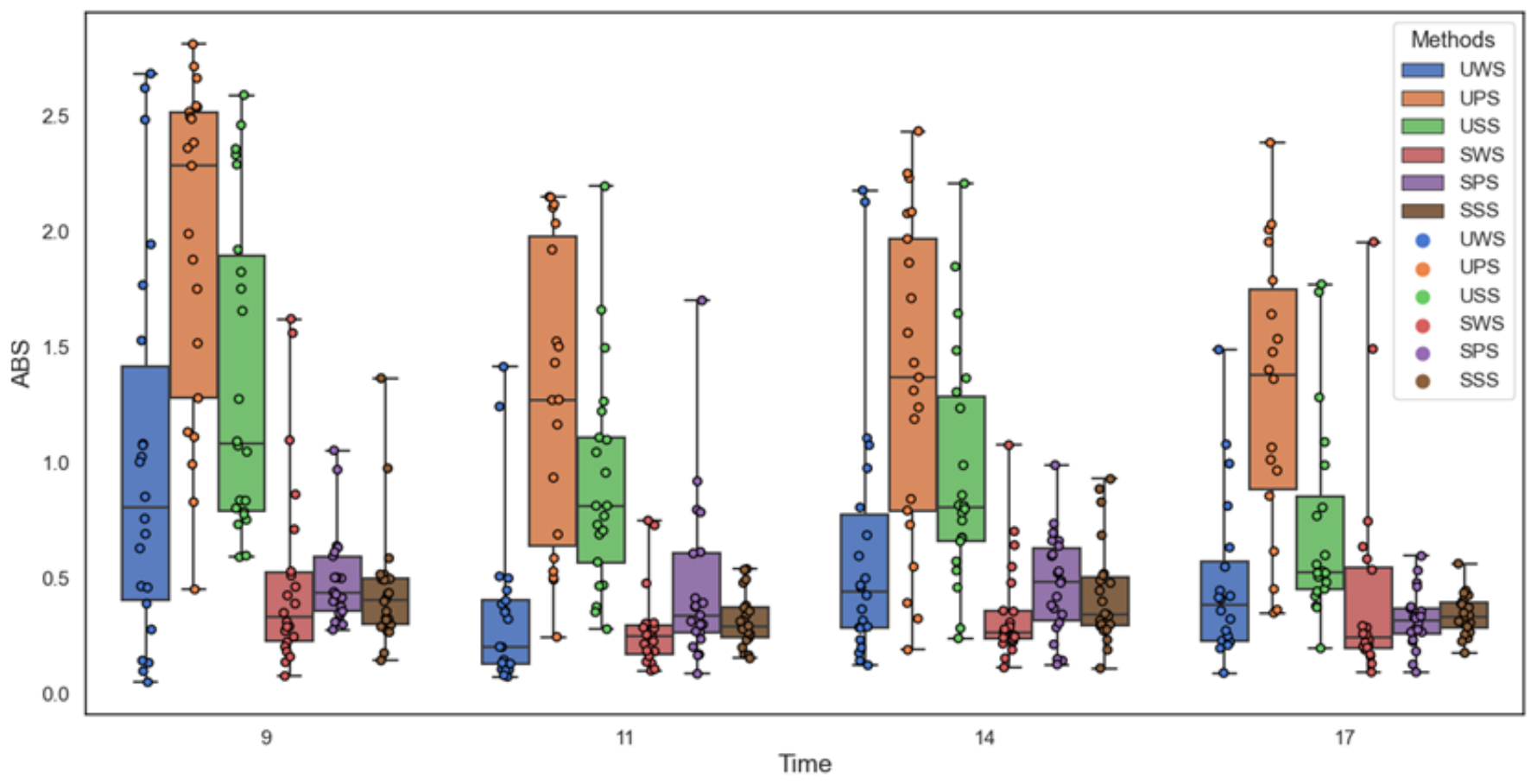

(a)
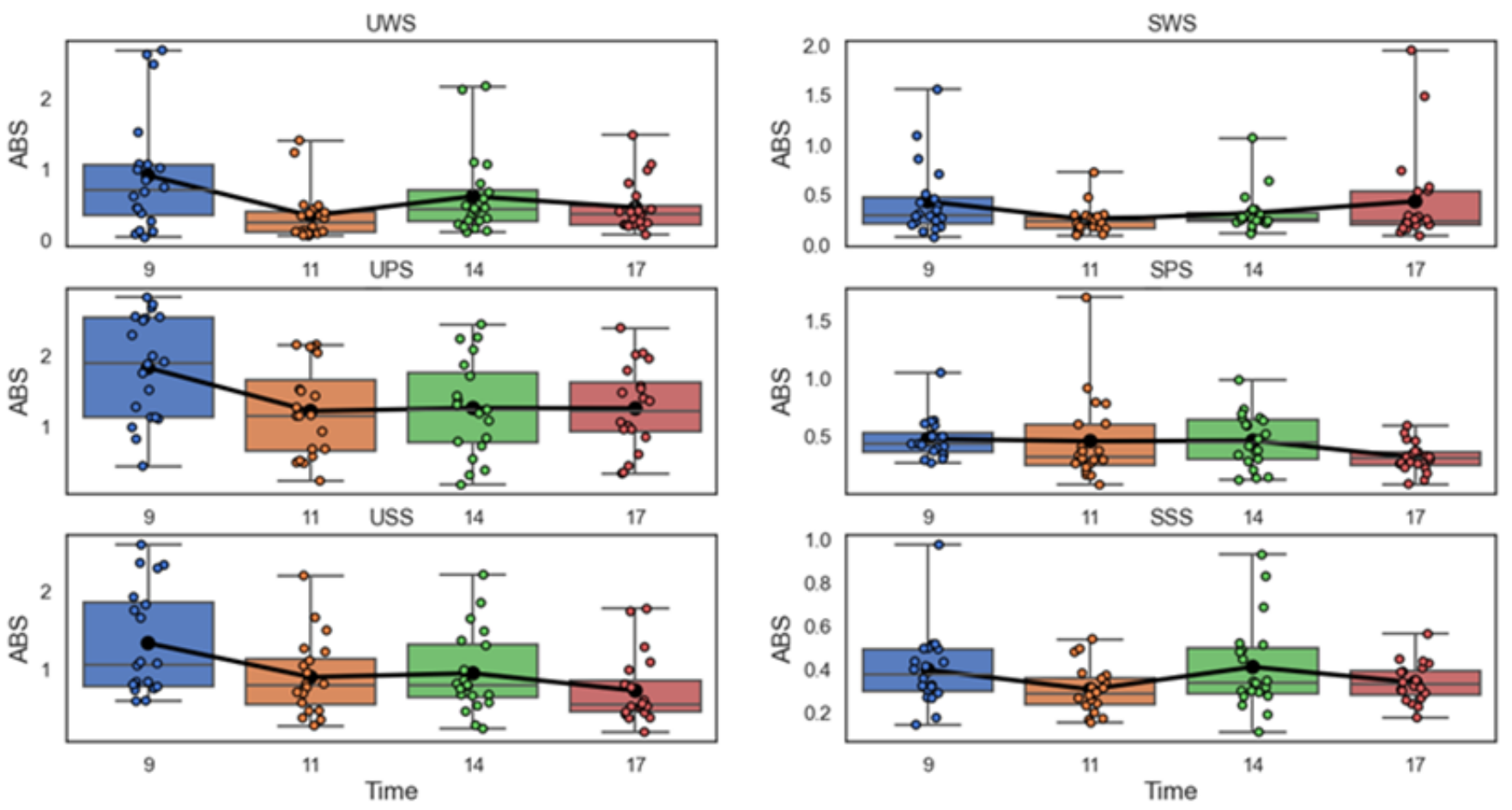

(b)

Figure 4

Saliva data summary, (a)ABS of saliva glucose concentration at different times and different saliva collection methods. (b). The trends of saliva glucose concentration with different saliva collection methods at different times. 

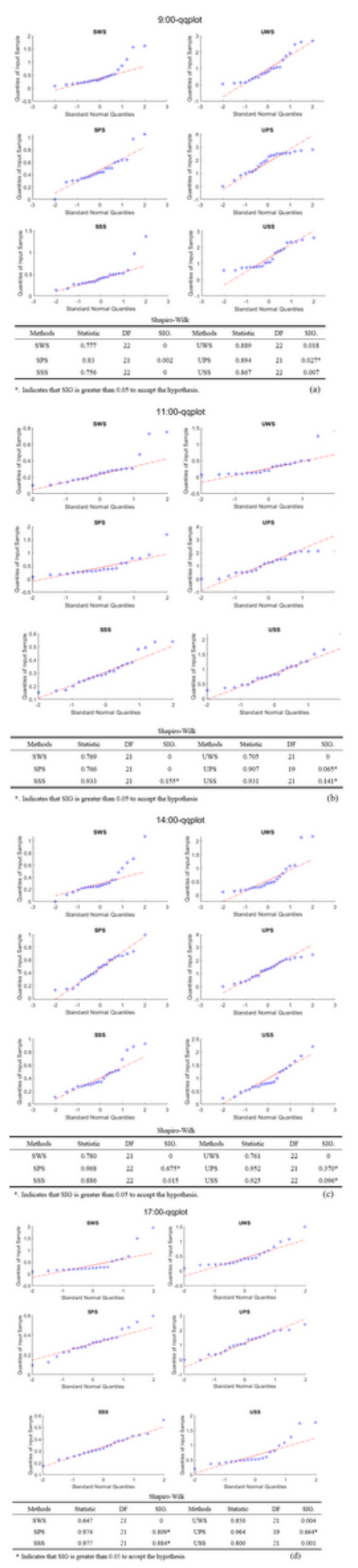

Figure 5

The normal distribution curve of different time and different collection methods. (a) The normal distribution curve of 9:00, (b) The normal distribution curve of 11:00, (c) The normal distribution curve of 14:00, (d) The normal distribution curve of 17:00. 

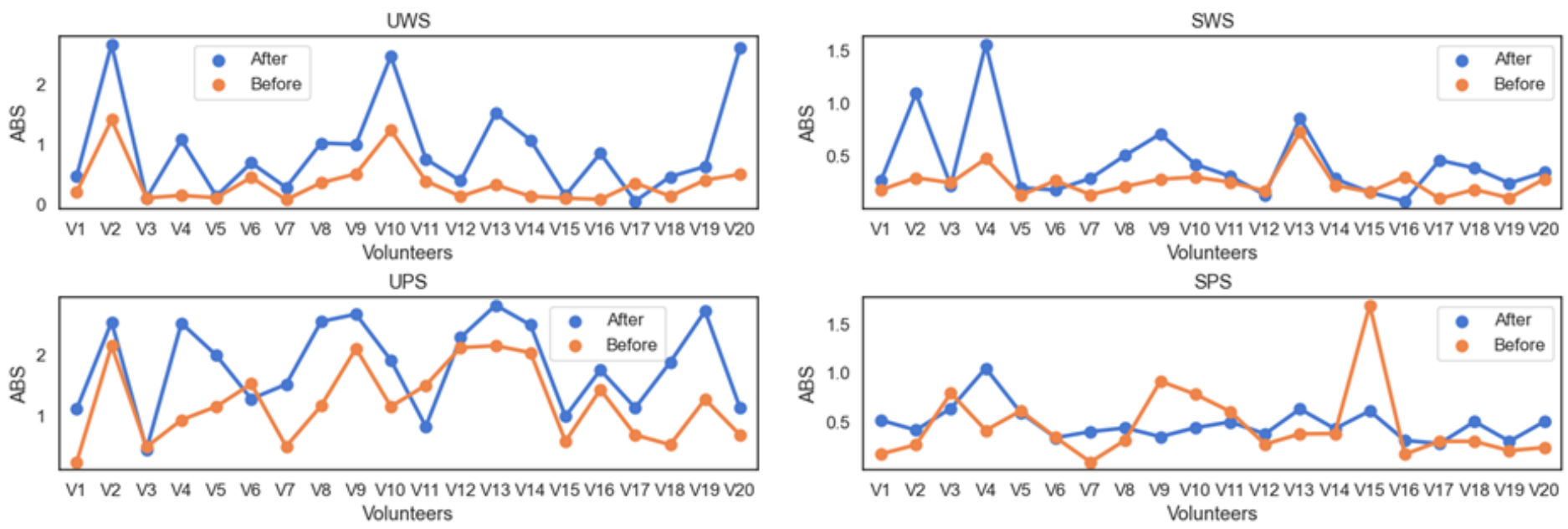

USS
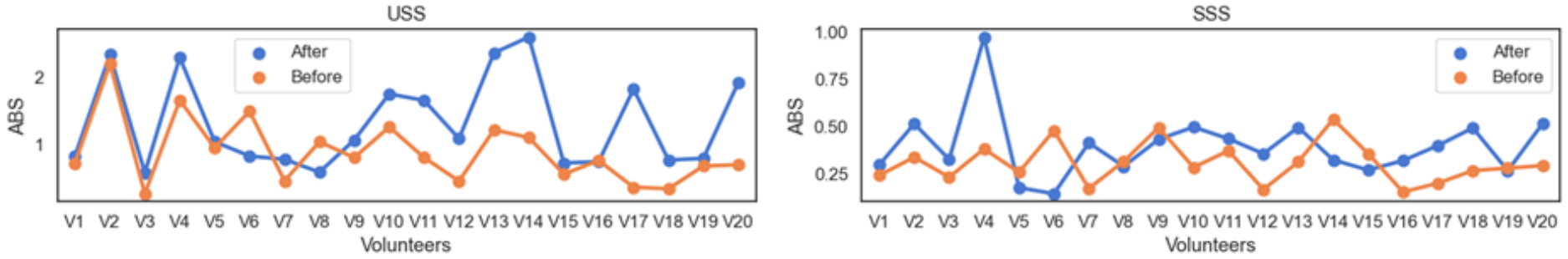

\section{Figure 6}

Fluctuations of saliva glucose before and after meals by different methods of saliva collection.

\section{Supplementary Files}

This is a list of supplementary files associated with this preprint. Click to download.

- Supplementarymaterial.doc 